\title{
UTILIZAÇÃO DE DIFERENTES MODELOS DE FRACIONAMENTO CORPORAL COMO CRITÉRIO DE REFERÉNCIA
}

\author{
Evaldo Maciel Pinto ${ }^{1,2}$, Diogo Guimarães ${ }^{3}$, Athos Henrique Rocha Villaça ${ }^{4}$, Sebastião Lobo da Silva ${ }^{1}$, Bibiano Madrid,6. \\ José Fernando Vila Nova de Moraes $^{1}$ - Marcelo Guido ${ }^{2}$ \\ 1.Doutorando em Educação Física - Universidade Católica de Brasília - Brasília - DF. \\ 2.Mestre em Educação Física - Universidade Católica de Brasília - Brasília - DF.
}

\begin{abstract}
RESUMO: A composição corporal tem importância fundamental para profissionais da nutrição, medicina e educação física. A existência de vários métodos e técnicas para mensuração dos componentes do organismo torna necessário um aprofundamento maior sobre suas origens, pressupostos e validação para utilização de forma adequada em populações de raças, idades e sexos específicos. Os modelos de fracionamento corporal são capazes de analisar, separadamente, a contribuição dos componentes na constituição do corpo humano, sendo utilizados frequentemente como critério referência para a validação de novos equipamentos. Desta forma, este artigo tem como objetivo reunir trabalhos científicos que utilizaram modelos de dois, três e quatro componentes para a determinação da composição corporal em diferentes populações, sendo eles como critérios de referência ou comparados entre si.

Palavras-chaves: composição corporal; modelos de fracionamento corporal; massa corporal.

BODY COMPARTMENT MODELS USED AS CRITERION METHOD

ABSTRACT: Body composition is of extreme importance to nutritionists, physicians and physical educators. The existence of a large variety of methods and techniques for body assessment makes it necessary to learn more about its origins, assumptions and validation, for proper use in populations with different ages, sex and ethnicity. Body compartment models are capable of analyzing, separately, the contribution of the components that are part of the human body, being frequently used as criterion methods for validation of new equipments. The purpose of this article is to gather research papers that utilized two-, three-, and four-compartment models for body assessment in different populations, being used as criterion methods or compared within themselves.
\end{abstract}

Keywords: body composition assessment; body compartment models; body mass.

Aceito em: 25/02/2011 - Revista de Educação Física 2012 Ago; 155:14-18. Rio de Janeiro - Brasil

\section{INTRODUÇÃO}

A composição corporal tem sido objetivo de estudo de pesquisadores há mais de 100 anos. Sua importância é fundamental para diversas áreas da saúde, como nutrição, algumas especialidades médicas e educação física'. Desta forma, a busca por métodos capazes de mensurar os elementos que compõem o corpo humano continua sendo frequentemente estudada por inúmeros pesquisadores.

Siri $^{2}$ definiu o corpo humano como sendo constituído, fundamentalmente, por quatro componentes: água, gordura, proteínas e minerais. Tais componentes poderiam sofrer variações de acordo com a raça, idade, sexo, hábitos nutricionais, prática de exercícios físicos, entre outros.

Sabido os componentes que constituem o organismo humano, o próximo passo foi elaborar técnicas capazes de mensurar os elementos in vivo. Porém, para se estabelecerem valores de referência, foram necessárias análises em cadáveres (chamados homens de referência), e, a partir daí foram estipuladas algumas constantes e criadas equações para cálculo e quantificação dos componentes corporais. Assim, surgiram os modelos de fracionamento corporal.

O primeiro modelo de fracionamento corporal criado dividia o corpo humano em dois componentes: massa gorda e massa livre de gordura. Neste modelo, foram estipulados valores constantes para a densidade da massa gorda e da massa livre de gordura, $0.9007 \mathrm{~g} /$ $\mathrm{cm}^{3}$ e $1.1000 \mathrm{~g} / \mathrm{cm}^{3}$, respectivamente. A partir daí, Siri (1956) e Bro $\square$ ek (1963) criaram fórmulas para o cálculo do percentual de gordura corporal através da mensuração da densidade do corpo ${ }^{3}$.

Este modelo sofreu diversas críticas, pois assumia as densidades da massa gorda e livre de gordura como valores fixos por todo o período de vida. $E$, além disso, estipulava que na massa gorda não existia água e a massa livre de gordura era composta por $73,2 \%$ de água. Desta forma, $\mathrm{Siri}^{2}$, ao verificar que tais premissas não eram adequadas para indivíduos de raças, idades e sexos diferentes, criou um novo modelo de composição corporal adicionando um terceiro componente, a água, criando o modelo de três componentes. Neste, o corpo humano era constituído por: massa gorda, água e massa seca livre de gordura e era assumida uma constante de 0,35 para a razão dos componentes mineral-proteína. Tal divisão aumentou a acurácia das mensurações por 
individualizar a quantidade de água nos sujeitos, porém, continuou estipulando constantes que sabidamente variam entre sexo, idade e raça $(3,4)$.

Com a possibilidade de se mensurar dois dos quatro componentes propostos por $\mathrm{Siri}^{2}$, restava apenas o desafio de quantificar mais um elemento para se obter a quantidade total de cada um dos componentes corporais. $\mathrm{O}$ avanço da tecnologia e o desenvolvimento de novos equipamentos como a Absortometria Radiológica de Dupla Energia (DEXA), tornou possível determinar o conteúdo mineral ósseo do corpo humano. Essa descoberta abriu portas para a criação de mais um modelo de fracionamento corporal, o de quatro componentes. Agora, era possível mensurar a densidade corporal, a água total do corpo e o conteúdo mineral ósseo5. Assim, o modelo de quatro componentes dependia de menos suposições e era capaz de individualizar ainda mais a parcela que cada elemento contribuía para a massa corporal total.

Na tabela abaixo podemos observar algumas fórmulas para estimar o percentual de gordura corporal a partir dos três modelos de fracionamento corporal propostos3.

Tabela 1. Equações para medir o percentual de gordura a partir dos modelos de dois, três e quatro componentes de composição corporal.

\begin{tabular}{|l|l|}
\hline Modelo & Equação \\
\hline Dois Componentes & \%Gordura $=\left(\frac{4.95}{\text { Dens }}-4.50\right) 100$ \\
\cline { 2 - 2 } & \%Gordura $=\left(\frac{4.570}{\text { Dens }}-4.142\right) 100$ \\
\hline Três Componentes & \%Gordura $=\left(\frac{2.118}{\text { Dens }}-0.78 \mathrm{~W}-1.354\right) 100$ \\
\cline { 2 - 2 } & \%Gordura $=\left(\frac{6.386}{\text { Dens }}-3.96 \mathrm{M}-6.090\right) 100$ \\
\hline Quatro Componentes & \%Gordura $=\left(\frac{\overline{\text { Dens }}}{}-0.714 \mathrm{~W}+1.146 \mathrm{~B}-2.0503\right) 100$ \\
\hline
\end{tabular}

Estes modelos de fracionamento corporal são extensivamente utilizados como padrão de referência para validação de outros instrumentos de mensuração da gordura corporal. Desta forma, o objetivo deste estudo foi o de reunir artigos científicos que utilizaram os modelos de dois, três e quatro componentes para a determinação da composição corporal em diferentes populações de diferentes idades, sendo eles utilizados como critérios de referência ou comparados entre si.

\section{METODOLOGIA}

Foram pesquisados artigos científicos de revistas internacionais em bancos de dados eletrônicos como o Periódicos Capes, Ebsco, Highwire e Pubmed. As palavras-chaves que fizeram parte da pesquisa foram: body compartment model; 2-compartment model; 3-compartment model; 4-compartment model em associação à body composition; body assessment e body composition assessment. As referências encontradas foram subdivididas em três tópicos distintos, de acordo com o modelo de fracionamento utilizado. $\mathrm{O}$ levantamento das referências bibliográficas ocorreu entre agosto e setembro de 2010.

\section{REVISÃO DE LITERATURA Modelo de dois componentes}

O modelo de dois componentes se mostra como o menos confiável por assumir constantes para as densidades de massa gorda e livre de gordura2. Um estudo comparando três diferentes métodos de mensuração de gordura corporal baseado no modelo de fracionamento de dois componentes mostrou boa correlação entre as técnicas $(r=0.90)$ e melhor sensibilidade para a classificação de sobrepesados e obesos do que o índice de massa corporal (IMC) ${ }^{6}$.

O modelo de doi componentes também mostrou resultados satisfatórios na monitoração da perda de peso em um grupo de obesos submetidos à restrição calórica7. Porém, quando comparado aos outros modelos notase uma diferença significativa nos resultados, podendo subestimar ou superestimar o percentual de gordura da população estudada. Em estudo com atletas de raça negra, o modelo de dois componentes subestimou significativamente o percentual de gordura em relação ao modelo de quatro componentes ${ }^{8}$.

Em uma amostra de adultos jovens e idosos, o modelo de dois componentes o obteve médias significativamente diferentes do modelo de quatro componentes nas duas populações ${ }^{9,10}$. O mesmo pode ser observado em pacientes portadores de cirrose hepática ${ }^{11}$. Os autores atribuíram tais diferenças às constantes assumidas pelo modelo em questão, pois, 
sabe-se que indivíduos com idades mais avançadas possuem menor densidade e hidratação da massa livre de gordura e pacientes com cirrose hepática sofrem anormalidades na homeostase e compartimentalização dos fluidos, no metabolismo de proteínas e na mineralização óssea ${ }^{(9-11)}$.

Ao comparar a composição corporal proveniente do modelo de dois componentes com os modelos de três e quatro componentes em homens e mulheres, observou-se uma superestimação do percentual de gordura relatado pelo modelo de dois componentes ${ }^{4}$. O mesmo foi descrito numa amostra com homens e mulheres atletas. Além disso, os valores encontrados para a densidade e hidratação da massa livre de gordura foram significativamente diferentes do preconizado pelas constantes $\left(1.100 \mathrm{~g} / \mathrm{cm}^{3} \text { e } 73,2 \% \text {, respectivamente }\right)^{12}$.

Um estudo com mulheres gestantes, em dois momentos, o primeiro em estágio avançado de gravidez e o segundo duas semanas após o parto, indicou que o modelo de dois componentes obteve erro significativo no percentual de gordura, nas duas etapas, quando comparado aos modelos de três e quatro componentes ${ }^{13}$.

\section{Modelo de três componentes}

O modelo de fracionamento corporal de três componentes traz como inovação a capacidade de individualizar a água corporal total. Um estudo com idosos do México utilizou este modelo para mensurar a quantidade de gordura corporal empregando as técnicas de pletismografia, para determinação da densidade corporal, e a técnica de diluição de isótopos, para água corporal total. Os resultados demonstraram que a pletismografia teve precisão e acurácia em ambos os sexos e que o percentual de gordura a partir da água corporal total não obteve diferenças significativas quando comparadas ao modelo de três componentes ${ }^{14}$.

$\mathrm{Na}$ avaliação da composição corporal em obesos, o modelo de três componentes surge como uma alternativa ao modelo de quatro componentes devido ao fato de equipamentos como o DEXA e a ativação de nêutrons não comportarem esses indivíduos. Porém, o modelo esbarra na utilização da constante da razão mineralproteína ${ }^{15}$.

Ao analisar métodos de laboratório e de campo para determinação da gordura corporal em universitários e fazendo comparações com 0 modelo de três componentes, observou-se que os equipamentos laboratoriais (pletismografia e pesagem hidrostática) produziram valores mais próximos de percentual de gordura. Porém, na ausência destes, a bioimpedância elétrica mostrou-se como uma técnica aceitável para esta população ${ }^{16}$.

Ao serem utilizados os diagnósticos de IMC para sobrepeso e obesidade e o modelo de três componentes para determinação de gordura corporal em indivíduos brancos, negros, hispânicos e asiáticos, constatou-se que apenas os asiáticos apresentavam maior quantidade de gordura corporal para o mesmo IMC em relação às outras raças ${ }^{17}$.

\section{Modelo de quatro componentes}

O Modelo de fracionamento corporal de quatro componentes é o mais utilizado como critério de referência por depender de um menor número de suposições para a determinação da gordura corporal. São inúmeros os artigos encontrados que procuram comparar e validar técnicas de composição corporal utilizando o modelo de quatro componentes como referência.

Heymsfield et al.18 comparou dois modelos de quatro componentes que utilizavam técnicas com custos diferenciados. No primeiro modelo, a composição corporal foi calculada utilizando DEXA, diluição de deutério e pesagem hidrostática, enquanto que o segundo modelo, utilizava métodos mais caros como ativação de nêutrons- $\square$ e análise de diluição de ${ }^{3} \mathrm{H} 2 \mathrm{O}$. Os resultados mostraram correlação significativa entre os métodos $(r=0,98 ; p<0.001)$ e as proporções representadas pelo conteúdo protéico, mineral e adiposo não obtiveram diferenças estatisticamente significativas.

A tentativa de validação de equações para bioimpedância elétrica em crianças, jovens e idosos de raça branca e negra em comparação com o modelo de quatro componentes mostrou que a bioimpedância subestimou a água corporal total e a massa livre de gordura em homens e mulheres negras e superestimou as mesmas variáveis em indivíduos brancos ${ }^{19}$. Já a antropometria, ao ser utilizada em população semelhante, foi capaz de subestimar ${ }^{20}$ ou superestimar em $10 \%$ o percentual de gordura ${ }^{21}$.

Em mulheres negras pré e pós-menopausadas foram encontradas mudanças siǵnificativas na composição corporal. Mulheres menopausadas revelaram água corporal total, conteúdo mineral ósseo e conteúdo protéico menor do que as pré-menopausadas. Apenas o 
percentual de gordura aumentou com a idade ${ }^{22}$.

DEXA subestimou o percentual de gordura corporal em adultos saudáveis quando comparado ao modelo de quatro componentes, a tendência para subestimação era aumentada em indivíduos mais magros ${ }^{23}$. Porém, ao ser analisado em idosos, não mostrou diferença para ambos os sexos ${ }^{24}$.

Em adultos jovens da Cingapura e Holanda foram utilizados o modelo de quatro componentes, a densitometria e a diluição de deutério óxido para quantificação da gordura corporal. Os componentes da massa livre de gordura não diferiram entre as mulheres dos dois países, porém, entre os homens, houve diferença significativa no conteúdo mineral e protéico. Em comparação com o modelo de quatro componentes, a diluição de deutério óxido, isoladamente, proporcionou resultados mais similares do que a densitometria25.

Em idosos, a pletismografia encontrou diferenças significativas em relação ao modelo de quatro componentes quando a amostra era separada por sexo. Porém, ao ser analisada toda a amostra essa diferença era suprimida26. A pesagem hidrostática superestimou o percentual de gordura dos homens mais magros e subestimou o dos mais obesos ${ }^{24}$. Já a água corporal total obteve valores com diferenças significativas em homens, mas não em mulheres, subestimando o percentual de gordura dos homens mais magros e superestimando o dos mais obesos ${ }^{24}$.

Em crianças, o percentual de gordura corporal calculado através da antropometria, DEXA, densitometria, diluição de isótopos e bioimpedância elétrica encontrou resultados, dentro do erro padrão de estimativa (2\%), apenas para a densitometria e a diluição de isótopos, quando comparados ao modelo de quatro componentes ${ }^{27}$. Outro estudo, com crianças obesas, utilizou DEXA, pletismografia e água corporal total e corrigiu os resultados pelo fator de correção de Lohman $^{28}$, encontrando diferenças significativas no percentual de gordura apenas para o DEXA ${ }^{29}$. Ellis et al.30 encontraram correlação alta e $R^{2}$ de 0,73 entre um aparelho de pletismografia específico para crianças de até sete quilos e o modelo de quatro componentes.

Outro estudo comparou, em crianças, o percentual de gordura a partir do DEXA, pesagem hidrostática, pletismografia e água corporal total em relação ao modelo de quatro componentes. Os resultados mostraram diferenças significativas na linha de identidade da regressão em todos os métodos utilizados, exceto a pletismografia e a pesagem hidrostática. Porém, ao ser analisado o erro padrão de estimativa, apenas a pletismografia não apresentou erro. Os autores atribuíram o erro elevado da pesagem hidrostática às constantes assumidas para a densidade da massa gorda e livre de gordura, que não são adequadas para crianças $^{31}$.

Um estudo com crianças pré-púberes e púberes demonstrou que o grau de hidratação da massa livre de gordura diminuiu após a puberdade $(75,7 \%$ e $74,4 \%$, respectivamente), o conteúdo mineral ósseo teve aumento $(4,9 \%$ e $5,0 \%)$, assim como a densidade da massa livre de gordura em meninos $(1.084 \mathrm{~g} / \mathrm{ml}-1.087 \mathrm{~g} /$ $\mathrm{ml})$ e em meninas $(1.086 \mathrm{~g} / \mathrm{ml}-1.091 \mathrm{~g} / \mathrm{ml}) 32$.

\section{CONCLUSÃO}

A evolução nos métodos e técnicas de composição corporal permitiu um melhor conhecimento sobre os elementos que constituem nosso organismo. O avanço da ciência tornou capaz a mensuração de componentes in vivo que antes só poderiam ser realizadas em cadáveres.

Seguindo a evolução tecnológica, os modelos de fracionamento corporal também evoluíram, e hoje dependem de poucas suposições para a determinação dos componentes corporais. Atualmente encontra-se uma vasta literatura que utilizam os modelos de dois, trêse quatro componentes para a mensuração da composição corporal, com este último modelo sendo considerado padrão-ouro. Não obstante, existem modelos de cinco1 e seis33 componentes que analisam de forma celular34 o organismo humano. Porém, tais modelos são pouco utilizados por dependerem de métodos extremamente técnicos e de custo elevado.

A utilização dos modelos de fracionamento corporal são de extrema importância na validação de novos equipamentos e equações para quantificação da composição corporal. O aperfeiçoamento dos métodos (para diferentes idades, sexo, raça, massa, etc.) permite uma melhor qualidade de pesquisa e acompanhamento nas mudanças dos conteúdos corporais da população.

\section{CONFLITOS DE INTERESSE}

Declaramos não haver conflitos de interesse. 


\section{REFERÊNCIAS BIBLIOGRÁFICAS}

1. Mutti D. Futsal da iniciação ao alto nível. São Paulo: Loyola, 1999.

2. Costa CF. Futsal aprenda ensinar. Florianópolis:

VisualBooks, 2003.

3. Santana WC de. Futsal: apontamentos pedagógicos na iniciação e na especialização. São Paulo: Autores associados, 2004.

4. Gomes AC, Machado JA. Futsal metodologia e planejamento na infância e adolescência. Londrina: Midiograf, 2001.

5. Magill RA. Motor learning: concepts and applications. $4^{\mathrm{a}}$ ed. São Paulo: Edgard Blücher, 2000.

6. Weineck J. Anatomia aplicada ao esporte. $3^{\mathrm{a}}$ ed. São Paulo: Manole, 1986.

7. Oliveira MC. Atletismo escolar: uma proposta de ensino na Educação infantil. Rio de Janeiro: Sprint, 2006.

8. Campos H. Capoeira na escola. Salvador: Presscolor, 1990.

9. Brasil - Secretaria de educação fundamental Parâmetros Curriculares Nacionais (PCNs): Educação Física / Brasília: MEC / SEF, 1998.

10. Barbanti VJ. Teoria e prática do treinamento desportivo. $2^{\mathrm{a}}$ ed. São Paulo: Edgard Brucher, 1997. 11. Panissa VLG. Exercício Concorrente. Analise do efeito agudo da ordem de execução sobre o gasto energético total: Rev Bras Med Esporte 2009;15(2):127131.

12. Hakkinen K, Alen M, Kraemer WJ, Gorostiaga

$\mathrm{E}$, Izquierdo $\mathrm{M}$, rusko $\mathrm{H}$, et al. Neuromuscular adaptations during concurrent strength and endurance training versus strength training. Eur J Appl Physiol 2003;89(1):42-52.

13. Paulo AC. Efeito do treinamento concorrente no desenvolvimento da força motora e da resistência aeróbia. Revista Mackenzie de Educação Física e Esporte - 2005;4(4):145-154.

14. Johnson BL, Nelson JK. Practical measurements for evaluation in physical education. Minnesota: Burgess Publishing Company, 1979.

15. Pitanga FJG. Testes, medidas e avaliação física e esportes. $5^{\mathrm{a}}$ ed. São Paulo: Phorte, 2008.

16. Passos WS. A influência do treinamento de futsal na velocidade e agilidade de escolares, EF Deportes 2009;13(129).

17. Nacif C, Moraes JM. Comparação da influência da capoeira na coordenação motora de escolares, EF Deportes 2010;14(137).

18. Martins SF. A contribuição da capoeira no desenvolvimento psicomotor de crianças entre seis e dez anos de idade. Apresentação de monografia à Universidade Candido Mendes como condição prévia para conclusão do curso de pós-graduação, rio de Janeiro, 2004.

19. Weineck J. Treinamento ideal. $9^{a}$ ed. São Paulo: Manole, 1999.

20. Venlioles FM. Escola de futebol. $2^{\mathrm{a}}$ ed. Rio de Janeiro: Sprint, 2004.
21. Melo RS. Jogos recreativos para futebol. $2^{\mathrm{a}}$ ed. Rio de Janeiro: Sprint, 2002.

\author{
Endereço pra correspondência: \\ Universidade Federal do Vale do São Francisco (UNIVASF) - \\ Colegiado de Educação Física (CEFIS) \\ Rua José de Sá Maniçoba, S/N \\ Centro - Petrolina/PE \\ CEP 56304-205 \\ e-mail dos autores: \\ josefernandomoraes@gmail.com / guidomss@yahoo.com.br
}

Telefone para contato: (61) 9151-6081 - José Fernando Vila Nova de Moraes 